PROCEEDINGS OF THE

AMERICAN MATHEMATICAL SOCIETY

Volume 35, No. 1, September 1972

\title{
EXISTENCE OF SOLUTIONS OF ABSTRACT MEASURE DIFFERENTIAL EQUATIONS
}

\author{
R. R. SHARMA
}

\begin{abstract}
In an earlier paper the author has introduced an abstract measure differential equation as a generalization of ordinary differential equations and measure differential equations, and proved a theorem for the existence and uniqueness of solutions of this equation. In the present paper the problem of the existence of solutions is further investigated.
\end{abstract}

1. Introduction. An abstract measure differential equation is defined in [3] as follows. Let $X$ be a linear space over the field $\mathscr{F}$ where $\mathscr{F}$ is the set $\boldsymbol{R}$ of real numbers or the set $\boldsymbol{C}$ of complex numbers. For each $x \in X$, define

$$
\begin{array}{lll}
S_{x}=\{\alpha x:-\infty<\alpha<1\}, & \bar{S}_{x}=\{\alpha x:-\infty<\alpha \leqq 1\} & \text { if } \mathscr{F}=\boldsymbol{R} ; \\
S_{x}=\{\alpha x: 0<|\alpha|<1\}, & \bar{S}_{x}=\{\alpha x: 0 \leqq|\alpha| \leqq 1\} & \text { if } \mathscr{F}=\boldsymbol{C} .
\end{array}
$$

Let $S \subset X$ where if $S$ is a proper subset of $X$, it is of the form $S_{\xi}$ for some $\xi \in X$. Let $\mathscr{M}$ be a $\sigma$-algebra in $S$ containing the sets $\bar{S}_{x}$ for all $x \in S$. We shall denote by $\operatorname{ca}(S, \mathscr{M})$ the space of all countably additive scalar functions (i.e. real measures or complex measures) on. $\mathscr{U}$. Let $\Omega \subset \mathscr{F}$ be defined by

$$
\Omega=\{\alpha:|\alpha|<a\} .
$$

Abstract measure differential equations are equations of the form

$$
d \lambda / d \mu=f\left(x, \lambda_{1}\left(\bar{S}_{x}\right)\right)
$$

where $\mu$ is a positive $\sigma$-finite measure or a complex measure on $\mathscr{M}$, $d \lambda / d \mu$ is Radon-Nikodym derivative of a measure $\lambda \in \operatorname{ca}(S, \mathscr{M})$ with respect to $\mu$, and $f$ is a function defined on $S \times \Omega$ such that $f\left(x, \lambda\left(S_{x}\right)\right)$ is $\mu$-integrable on $S$ for each $\lambda \in \operatorname{ca}(S, \mathscr{M})$.

The solution of $(*)$ is defined as follows. Let $\alpha_{0} \in \Omega, x_{0} \subset S, S_{\alpha_{0}}=X_{0} \in \mathscr{M}$ and let $\mathscr{M}_{0}$ be the smallest $\sigma$-algebra in $X_{0}$ containing $\bar{S}_{x_{0}}-S_{x_{0}}$ and the sets $\bar{S}_{x}$ for $x \in X_{0}-S_{x_{0}}$. A measure $\lambda \in \operatorname{ca}\left(X_{0}, \mathscr{M}_{0}\right)$ is called a solution of $(*)$

Received by the editors September 21, 1971 and, in revised form, November 24, 1971.

AMS 1970 subject classifications. Primary 34G05; Secondary 46G99.

Key words and phrases. Abstract measure differential equation, real measures, complex measures, Radon-Nikodym derivative, total variation measure, pseudometric. 
on $X_{0}$ with initial data $\left[\bar{S}_{x_{0}}, \alpha_{0}\right]$ (to be denoted by $\lambda\left[X_{0} ; S_{x_{0}}, \alpha_{0}\right]$ ) if $\lambda\left(S_{x_{0}}\right)=$ $\alpha_{0}, \lambda(E) \in \Omega$ for $E \in \mathscr{M}_{0}, \lambda \ll \mu$ on $X_{0}-S_{x_{0}}$ and $\lambda$ satisfies $(*)$ a.e. $[\mu]$ on $X_{0}-S_{x_{0}}$.

An existence and uniqueness theorem is proved in [3] where one of the assumptions for $f$ is to satisfy a Lipschitz condition in $\alpha$. In the present paper the existence of a solution (without claiming its uniqueness) is established by changing some of the assumptions, particularly the Lipschitz condition hypothesis for $f$ is replaced by the continuity of $f$ in $\alpha$ for fixed $x$. Taking ca $(X, \mathscr{M})$ to be the space of real measures, maximum and minimum solutions are defined and their existence is demonstrated under the hypotheses of the existence theorem. It may be noted that Theorem 1 of this paper includes and extends [1, Theorem 1.1, p. 43] and Theorem 2 includes and extends [1, Theorem 1.2, p. 45].

2. Existence of solutions. For any $E_{1}, E_{2} \in \mathscr{M}$, we define

$$
\rho\left(E_{1}, E_{2}\right)=|\mu|\left(E_{1}-E_{2}\right)+|\mu|\left(E_{2}-E_{1}\right)
$$

where $|\mu|$ denotes the total variation measure of $\mu$. It is clear that for any $E_{1}, E_{2} \in \mathscr{M}$,

$$
\begin{aligned}
& \rho\left(E_{1}, E_{2}\right) \geqq 0, \quad \rho\left(E_{1}, E_{2}\right)=0 \quad \text { if } E_{1}=E_{2}, \\
& \rho\left(E_{1}, E_{2}\right)=\rho\left(E_{2}, E_{1}\right) .
\end{aligned}
$$

Also, for any $E_{1}, E_{2}, E_{3} \in \mathscr{M}$, we have

$$
\rho\left(E_{1}, E_{2}\right) \leqq \rho\left(E_{1}, E_{3}\right)+\rho\left(E_{3}, E_{2}\right),
$$

which follows from

$$
\begin{aligned}
& \left(E_{1}-E_{2}\right) \subset\left(E_{1}-E_{3}\right) \cup\left(E_{3}-E_{2}\right), \\
& \left(E_{2}-E_{1}\right) \subset\left(E_{3}-E_{1}\right) \cup\left(E_{2}-E_{3}\right) .
\end{aligned}
$$

The function $\rho$ thus defines a pseudometric for $\mathscr{M}$.

We shall now prove the following existence theorem.

THEOREM 1. Let $\alpha_{0} \in \Omega, x_{0} \in S$ and let for each $\xi \in S-S_{x_{0}}$ the smallest $\sigma$-algebra containing $S_{x_{0}}-S_{x_{0}}$ and $S_{x}, x \in S_{\xi}-S_{x_{0}}$, be compact in the topology generated by the pseudometric $\rho$ defined by (2.1). Then there exists a solution $\lambda_{0}=\lambda_{0}\left[S_{x^{\prime}} ; S_{x_{0}}, \alpha_{0}\right]$ of $(*)$ for some $x^{\prime} \in S-S_{x_{0}}$ if the following conditions are satisfied:

(i) $\mu\left(S_{x_{0}}\right) \neq 0$ and $|\mu|\left(S_{x}-S_{x}\right)=0$ for each $x \in S-S_{x_{0}}$;

(ii) there exists a $\mu$-integrable function $w$ on $S$ such that $|f(x, \alpha)| \leqq w^{\prime}(x)$ uniformly in $\alpha \in \Omega$;

(iii) $f$ is continuous in $\alpha$ for each fixed $x$. 
Proof. Choose a real number $r>1$ such that

$$
\int_{\bar{s}_{r x_{0}}-s_{x_{0}}} w(x) d|\mu|<a-\left|\alpha_{0}\right| .
$$

It is possible to choose such a real number $r$ (see the proof of Theorem 1 in [3]).

Let $\mathscr{M}_{0}$ be the smallest $\sigma$-algebra containing $\bar{S}_{x_{0}}-S_{x_{0}}$ and all the sets of the form $\bar{S}_{x}$ for $x \in \bar{S}_{r x_{0}}-S_{x_{0}}$. In what follows, it will always be assumed that $E \in \mathscr{M}_{0}$. Define measures $\lambda_{j}$ by

$$
\begin{aligned}
\lambda_{j}(E) & =\alpha_{0} & & \text { for } E=\bar{S}_{x_{0}}, \\
& =0 & & \text { for } E \subset \bar{S}_{x_{0}+\xi j}-S_{x_{0}}, \\
& =\int_{E} f\left(x, \lambda_{j}\left(\bar{S}_{x-\xi_{j} j}\right)\right) d \mu & & \text { for } E \subset \bar{S}_{r x_{0}}-S_{x_{0}+\xi_{j}},
\end{aligned}
$$

where

$$
\xi_{j}=[(r-1) / j] x_{0} .
$$

Then $\lambda_{1}$ is defined by the first two expressions in (2.3). For any fixed $j \geqq 2$, the first and second expressions in (2.3) define $\lambda_{j}$ for $E \subset S_{x_{0}+\xi_{j}}$; and since $(x, 0)$ and $\left(x, \alpha_{0}\right) \in \bar{S}_{r x_{0}} \times \Omega$, the last expression defines $\lambda_{j}$ for $E \subset \bar{S}_{x_{0}+2 \xi_{j}}-S_{x_{0}+\xi_{j}} . \lambda_{j}$ is then defined for $E \subset S_{x_{0}+2 \xi_{j}}$. Also, for $E \subset S_{x_{0}+2 \xi_{j}}$, we have

$$
\left|\lambda_{j}(E)\right| \leqq\left|\alpha_{0}\right|+\int_{\bar{S}_{r x_{0}}-S_{x_{0}}} w(x) d|\mu|<a,
$$

by condition (ii) and (2.2); and, therefore,

$$
\lambda_{j}(E) \in \Omega .
$$

Assume that $\lambda_{j}$ is defined for $E \subset \bar{S}_{x_{0}+k \xi_{j}}$ when $2 \leqq k<j$. Then the last expression in (2.3) defines $\lambda_{j}$ for $\bar{S}_{x_{0}+(k+1) \xi_{j}}-S_{x_{0}+k_{\xi}}$ and thus $\lambda_{j}$ is defined for all $E \subset \bar{S}_{x_{0}+(k+1) \xi_{j}}$. Also, for such $E$ 's, $\lambda_{j}(E)$ satisfies $(2.5)$ and hence (2.6) because of condition (ii) and (2.2). Therefore, by induction, (2.3) defines $\lambda_{j}$ on $\mathscr{M}_{0}$.

Let $E_{1}, E_{2} \in \mathscr{M}_{0}$ be such that $\rho\left(E_{1}, E_{2}\right)<|\mu|\left(S_{x_{0}}\right)$. Then $S_{x_{0}}$ is a subset either of both $E_{1}$ and $E_{2}$ or of neither of $E_{1}$ and $E_{2}$. For, otherwise,

$$
\rho\left(E_{1}, E_{2}\right)=|\mu|\left(E_{1}-E_{2}\right)+|\mu|\left(E_{2}-E_{1}\right) \geqq|\mu|\left(S_{x_{0}}\right) .
$$

It can be verified that either

$$
\lambda_{j}\left(E_{1}\right)-\lambda_{j}\left(E_{2}\right)=0
$$


or else

$$
\lambda_{j}\left(E_{1}\right)-\lambda_{j}\left(E_{2}\right)=\int_{E_{1}-E_{2}} f\left(x, \lambda_{j}\left(\bar{S}_{x-\xi_{j}}\right)\right) d \mu-\int_{E_{2}-E_{1}} f\left(x, \lambda_{j}\left(\bar{S}_{x-\xi_{j}}\right)\right) d \mu,
$$

and therefore, by condition (ii),

$$
\begin{aligned}
\left|\lambda_{j}\left(E_{1}\right)-\lambda_{j}\left(E_{2}\right)\right| & <\int_{E_{1}-E_{2}} w(x) d|\mu|+\int_{E_{2}-E_{1}} w(x) d|\mu| \\
& =\int_{\left(E_{1}-E_{2}\right) \cup\left(E_{2}-E_{1}\right)} w(x) d|\mu| .
\end{aligned}
$$

By Dunford and Schwartz [2, Theorem 20, p. 114], if

$$
\nu(E)=\int_{E} w(x) d|\mu|
$$

then

$$
\lim _{|\mu|(E) \rightarrow 0} v(E)=0
$$

This implies that the function $v$ defined by (2.8) is continuous (and hence uniformly continuous) on the compact pseudometric space $\mathscr{M}_{0}$. From the uniform continuity of $v$ on $\mathscr{M}_{0}$, it follows from (2.7) that given any $\varepsilon>0$, there exists a $\delta(\varepsilon), 0<\delta\left(<|\mu|\left(S_{x_{0}}\right)\right)$ such that $\left|\lambda_{j}\left(E_{1}\right)-\lambda_{j}\left(E_{2}\right)\right|<\varepsilon$ whenever $|\mu|\left(E_{1}-E_{2}\right)+|\mu|\left(E_{2}-E_{1}\right)=\rho\left(E_{1}, E_{2}\right)<\delta$; i.e. $\left\{\lambda_{j}\right\}$ is an equicontinuous set. Moreover, $\left\{\lambda_{j}\right\}$ is uniformly bounded since, by (2.5), we have

$$
\sup _{E \in, \mathscr{M}_{0}}\left|\hat{\lambda}_{j}(E)\right| \leqq\left|\alpha_{0}\right|+\int_{\tilde{S}_{r x_{0}}-S_{x_{0}}} w(x) d|\mu|<a .
$$

Therefore, it follows by the Arzela-Ascoli theorem $\left[2\right.$, p. 266] that $\left\{\lambda_{j}\right\}$ is conditionally compact in the space $C\left(\mathscr{M}_{0}\right)$ of all bounded continuous scalar functions on $\mathscr{H}_{0}$. Hence there exists a subsequence $\left\{\lambda_{j_{k}}\right\}$ of $\left\{\lambda_{j}\right\}$ and a function $\lambda_{0} \in C\left(\mathscr{M}_{0}\right)$ such that $\lambda_{j_{k}} \rightarrow \lambda_{0}$ uniformly on $\mathscr{M}_{0}$ as $k \rightarrow \infty$. And since

$$
\rho\left(\bar{S}_{x-\xi_{k}}, \bar{S}_{x}\right)=|\mu|\left(\bar{S}_{x}-\bar{S}_{x-\xi_{j_{k}}}\right) \rightarrow|\mu|\left(\bar{S}_{x}-S_{x}\right)=0,
$$

by condition (i) it follows that $\lambda_{j_{k}}\left(\bar{S}_{x-\xi_{j_{k}}}\right) \rightarrow \lambda_{0}\left(\bar{S}_{x}\right)$. Also, by (2.9), $\lambda_{0}(E) \in \Omega$ for $E \in \mathscr{M}_{0}$. Hence the continuity of $f$ in $\alpha$ for fixed $x$ (condition (iii)) implies

$$
\lim _{k \rightarrow x} f\left(x, \lambda_{j_{k}}\left(\bar{S}_{x-j_{j}}\right)\right)=f\left(x, \lambda_{0}\left(\bar{S}_{x}\right)\right)
$$

From (2.10) and condition (ii), it follows by Lebesgue's dominated convergence theorem that

$$
\lim _{l \rightarrow \infty} \int_{E} f\left(x, \lambda_{j_{k}}\left(\bar{S}_{x-\xi_{j}}\right)\right) d \mu=\int_{E} f\left(x, \lambda_{0}\left(\bar{S}_{x}\right)\right) d \mu
$$


Now replacing $j$ by $j_{k}$ in (2.3) and letting $k \rightarrow \infty$, we obtain

$$
\begin{aligned}
& \lambda_{0}(E)=\alpha_{0} \quad \text { for } E=\bar{S}_{x_{0}}, \\
& =\int_{E} f\left(x, \lambda_{0}\left(S_{x}\right)\right) d \mu \quad \text { for } E \subset S_{r x_{b}}-S_{x_{0}}
\end{aligned}
$$

Thus $\lambda_{0}$ satisfies $(*)$ a.e. $[\mu]$ on $S_{r x_{0}}-S_{x_{0}}$. It also follows from (2.11) that $\lambda_{0}$ is countably additive on $\mathscr{M}_{0}$. Hence $\lambda_{0}$ is a solution of $(*)$ on $S_{r x_{0}}$ with initial condition $\lambda_{0}\left(\bar{S}_{x_{0}}\right)=\alpha_{0}$. This completes the proof.

3. Maximum and minimum solutions. In this section we shall be concerned with real measures only and so $\operatorname{ca}(S, \mathscr{M})$ will now denote the space of real measures on $\mathscr{M}$. Let $\alpha_{0}, x_{0}, X_{0}$ and $\mathscr{M}_{0}$ be the same as in $\S 1$. Let $\Lambda$ be the set of all solutions of $(*)$ on $X_{0}$ with initial data $\left[\bar{S}_{x_{0}}, \alpha_{0}\right]$.

Definition. A maximum solution of $(*)$ on $X_{0}$ with initial data $\left[\bar{S}_{x_{0}}, \alpha_{0}\right]$ is a solution $\lambda_{1 Y} \in \Lambda$ with the property

$$
\lambda(E) \leqq \lambda_{M}(E) \quad\left(E \in \mathscr{M}_{0}\right)
$$

for each $\lambda \in \Lambda$. Similarly, $\lambda_{m} \in \Lambda$ will be called a minimum solution of (*) on $X_{0}$ with initial data $\left[S_{x_{0}}, \alpha_{0}\right]$ if

for each $\lambda \in \Lambda$.

$$
\lambda(E) \geqq \lambda_{m}(E) \quad\left(E \in \mathscr{M}_{0}\right)
$$

We shall now establish the existence of $\lambda_{M I}$ and $\lambda_{m}$ under the assumptions of Theorem 1.

THEOREM 2. Let the hypotheses of Theorem 1 be satisfied. Then there exist a maximum solution $\lambda_{M}$ and a minimum solution $\lambda_{m}$ of $(*)$ on $X_{0}=\bar{S}_{r x_{0}}\left(\bar{S}_{r x_{0}}\right.$ being defined by (2.2)) with initial data $\left[\bar{S}_{x_{0}}, \alpha_{0}\right]$.

Proof. Define $\lambda^{*}(E)=\sup _{i \in \Lambda}\{\lambda(E)\} \quad\left(E \in \mathscr{H}_{0}\right)$. We shall prove that $\lambda^{*}$ is a maximal solution $\lambda_{M}$.

Clearly $\lambda^{*}\left(\bar{S}_{x_{0}}\right)=\alpha_{0}$ and $\lambda^{*} \ll \mu$ on $X_{0}-S_{x_{0}}$. By condition (ii) of Theorem 1 , each $\lambda \in \Lambda$ satisfies the inequality

And, since

$$
|\lambda(E)|<\left|x_{0}\right|+\int_{\bar{s}_{r x_{0}}-S x_{0}} w(x) d|\mu| \quad\left(E \in \mathscr{H}_{0}\right)
$$

$$
\left|\lambda^{*}(E)\right|=\left|\sup _{\lambda \in \Lambda} \lambda(E)\right| \leqq \sup _{\lambda \in \Lambda}|\lambda(E)| \quad\left(E \in \mathscr{M}_{0}\right) .
$$

it follows from (3.1) and (2.2) that $\lambda^{*}(E) \in \Omega$ for each $E<. H_{0}$.

We shall now show that $\lambda^{*}$ satisfies $(*)$ a.e. $[\mu]$ on $X_{0}-S_{x_{0}}$. It can be shown, in the same way as the equicontinuity of $\left\{\hat{\lambda}_{j} ;\right.$ was demonstrated in the proof of Theorem 1 , that $\Lambda$ is an equicontinuous set. Thus, given any 
$\varepsilon>0$, there exists a $\delta=\delta(\varepsilon)>0$ such that $E_{1}, E_{2} \in \mathscr{M}_{0}$ and $\rho\left(E_{1}, E_{2}\right)<\delta$ imply

$$
\left|\lambda\left(E_{1}\right)-\lambda\left(E_{2}\right)\right|<\varepsilon \quad \text { for each } \lambda \in \Lambda
$$

and hence

$$
\begin{aligned}
\left|\lambda^{*}\left(E_{1}\right)-\lambda^{*}\left(E_{2}\right)\right| & =\left|\sup _{\lambda \in \Lambda} \lambda\left(E_{1}\right)-\sup _{\lambda \in \Lambda}\left(E_{2}\right)\right| \\
& \leqq\left|\sup _{\lambda \in \Lambda}\left(\lambda\left(E_{1}\right)-\lambda\left(E_{2}\right)\right)\right| \\
& \leqq \sup _{\lambda \in \Lambda}\left|\lambda\left(E_{1}\right)-\lambda\left(E_{2}\right)\right| \leqq \varepsilon .
\end{aligned}
$$

Let $x_{1}, x_{2}, \cdots, x_{n}=r x_{0}$ be such that

(3.4) $S_{x_{i-1}} \subset S_{x_{i}}$ and $\max |\mu|\left(S_{x_{i}}-S_{x_{i-1}}\right)<\delta, \quad(i=1,2, \cdots, n)$.

For the given $\varepsilon$, choose a $\lambda_{i} \in \Lambda$ for each $x_{i}(i=0,1, \cdots, n-1)$ so that

and for $i \geqq 1$

$$
0 \leqq \lambda^{*}\left(\bar{S}_{x_{i}}\right)-\lambda_{i}\left(\bar{S}_{x_{i}}\right) \leqq \varepsilon
$$

$$
\lambda_{i}\left(\bar{S}_{x_{i}}\right)-\lambda_{i-1}\left(S_{x_{i}}\right) \geqq 0 .
$$

This is possible from the definition of $\lambda^{*}$.

Now define a function $\lambda_{\varepsilon}$ as follows: Let $\lambda_{\varepsilon}(E)=\lambda_{n-1}(E)$ for $E \subset$ $S_{r x_{0}}-S_{x_{n-1}}\left(E \in \mathscr{M}_{0}\right)$. If $\lambda_{n-1}\left(\bar{S}_{x_{n-1}}\right)>\lambda_{n-2}\left(S_{x_{n-1}}\right)$, let $x_{n-2}^{\prime}$ (if it exists) be such that

$$
x_{n-2}^{\prime} \in S_{x_{n-1}}-S_{x_{n-2}}, \quad \lambda_{n-1}\left(S_{x_{n-2}^{\prime}}\right)=\lambda_{n-2}\left(S_{x_{n-2}^{\prime}}\right)
$$

and if $x_{n-2}^{\prime \prime}$ also satisfies these conditions then $S_{x_{n-2}^{\prime}} \supset \bar{S}_{x_{n-2}^{\prime \prime}}$. If such an $x_{n-2}^{\prime}$ does not exist, let $x_{n-2}^{\prime}=x_{n-2}$. If $\lambda_{n-1}\left(\bar{S}_{x_{n-1}}\right)=\lambda_{n-2}\left(\bar{S}_{x_{n-1}}^{n-2}\right)$, let $x_{n-2}^{\prime}=$ $x_{n-1}$. Define

$$
\begin{aligned}
\lambda_{\varepsilon}(E) & =\lambda_{n-1}(E) \quad \text { for } E \subset S_{x_{n-1}}-S_{x_{n-2}^{\prime}}, \\
& =\lambda_{n-2}(E) \quad \text { for } E \subset S_{x_{n-2}^{\prime}}-S_{x_{n-2}} ;
\end{aligned}
$$

and

$$
\begin{aligned}
\lambda_{\varepsilon}\left(\bar{S}_{r_{n-2}^{\prime}}\right)=\lambda_{n-2}\left(\bar{S}_{x_{n-2}^{\prime}}\right) \quad \text { when } x_{n-2}^{\prime} \in \bar{S}_{x_{n-1}}-S_{x_{n-2}}, \\
=\lambda_{n-1}\left(\bar{S}_{x_{n-2}^{\prime}}\right) \quad \text { when } x_{n-2}^{\prime}=x_{n-2} .
\end{aligned}
$$

If $\hat{\lambda}_{\varepsilon}\left(\bar{S}_{x_{n-2}}\right)>\hat{i}_{n-3}\left(\bar{S}_{x_{n-2}}\right)$, let $x_{n-3}^{\prime}$ (if it exists) $\in S_{x_{n-2}}-S_{x_{n-3}}$ be such that

$$
\begin{aligned}
\lambda_{n-3}\left(S_{r_{, 3}^{\prime},}\right) & =\lambda_{n-2}\left(\bar{S}_{r_{n-3}^{\prime}}\right) \quad \text { when } x_{n-2}^{\prime} \in \bar{S}_{r_{n-1}}-S_{x_{n-2}}, \\
& =\lambda_{n-1}\left(\bar{S}_{r_{n-3}^{\prime}}\right) \quad \text { when } x_{n-2}^{\prime}=x_{n-2} ;
\end{aligned}
$$


and if $x_{n-3}^{\prime \prime}$ be any point with this property then $\bar{S}_{x_{n-3}^{\prime}} \supset \bar{S}_{x_{n-3}^{\prime \prime}}$. If such an $x_{n-3}^{\prime}$ does not exist, let $x_{n-3}^{\prime}=x_{n-3}$. If $\lambda_{\varepsilon}\left(\bar{S}_{x_{n-2}}\right)=\lambda_{n-3}\left(S_{x_{n-2}}\right)$, let $x_{n-3}^{\prime}=x_{n-2}$. Define

$$
\begin{aligned}
\lambda_{\varepsilon}(E) & =\lambda_{n-1}(E) \quad \text { for } E \subset S_{x_{n-2}}-S_{x_{n-3}^{\prime}} \text { when } x_{n-2}^{\prime}=x_{n-2,} \\
& =\lambda_{n-2}(E) \text { for } E \subset S_{x_{n-2}}-S_{x_{n-3}^{\prime}} \text { when } x_{n-2}^{\prime} \in S_{x_{n-1}}-\bar{S}_{x_{n-2},}, \\
& =\lambda_{n-3}(E) \quad \text { for } E \subset S_{x_{n-3}^{\prime}}-S_{x_{n-3}}
\end{aligned}
$$

$$
\left(E \in \mathscr{M}_{0}\right)
$$

Continuing in this way a function $\lambda_{\varepsilon}$ can be defined on all sets $E\left(\in \mathscr{M}_{0}\right)$ such that $E$ is a subset of one of the sets

$$
S_{x_{i}^{\prime}}-S_{x_{i}}, \quad S_{x_{i+1}}-S_{x_{i}{ }^{\prime}}, \quad \bar{S}_{r x_{0}}-S_{x_{n-1}} \quad(i=0,1, \cdots, n-2) .
$$

Also, define $\lambda_{\varepsilon}\left(\bar{S}_{x_{0}}\right)=\alpha_{0}$. Now extend the definition of $\lambda_{\varepsilon}$ on $\mathscr{M}_{0}$ by countable-additivity; i.e. if $E\left(\in \mathscr{M}_{0}\right) \subset \bar{S}_{r x_{0}}-S_{x_{0}}$, define

$$
\begin{aligned}
\lambda_{\varepsilon}(E)= & \sum_{i=0}^{n-2} \lambda_{\varepsilon}\left(E \cap\left(S_{x_{i}{ }^{\prime}}-S_{x_{i}}\right)\right)+\sum_{i=0}^{n-2} \lambda_{\varepsilon}\left(E \cap\left(S_{x_{r_{1}}}-S_{x_{i}}{ }^{\prime}\right)\right) \\
& +\lambda_{\varepsilon}\left(E \cap\left(S_{r x_{0}}-S_{x_{n-1}}\right)\right) .
\end{aligned}
$$

Now it can be easily seen that $\lambda_{\varepsilon}$ is a solution in $\Lambda$ having the property:

$$
0 \leqq \lambda^{*}\left(\bar{S}_{x_{i}}\right)-\lambda_{\varepsilon}\left(\bar{S}_{x_{i}}\right) \leqq \varepsilon \quad(i=0,1, \cdots, n)
$$

Furthermore, if $E\left(\in \mathscr{M}_{0}\right)$ is a subset of one of the sets in (3.5), it follows from (3.2), (3.3), (3.4) and (3.7) that

$$
0 \leqq \lambda^{*}(E)-\lambda_{\varepsilon}(E)<3 \varepsilon .
$$

Letting $\varepsilon=1 / m(m=1,2, \cdots)$, we obtain a sequence $\lambda_{1 / m}$ of solutions such that

$$
\lim _{m \rightarrow \infty} \lambda_{1 / m}(E)=\lambda^{*}(E)
$$

where $E\left(\epsilon \cdot \mathscr{H}_{0}\right)$ is a subset of one of the sets in (3.5). For $E\left(\in \mathscr{M}_{0}\right) \subset$ $\bar{S}_{r x_{0}}-S_{x_{0}}$, we have

$$
\begin{aligned}
& \lambda^{*}(E) \geqq \lim _{m \rightarrow \infty} \lambda_{1 / m}(E)=\sum_{i=0}^{n-2} \lambda^{*}\left(E \cap\left(S_{r_{i}^{\prime}}-S_{s^{\prime}}\right)\right) \\
& +\sum_{i=0}^{n=2} \lambda^{*}\left(E \cap\left(S_{s_{i+1}}-S_{x_{i}}\right)\right)+\lambda^{*}\left(E \cap\left(\bar{S}_{,_{1}}-S_{r_{n-1}}\right)\right),
\end{aligned}
$$


by (3.6) and (3.9). Also,

$$
\begin{aligned}
\lambda^{*}(E)= & \sup _{i=\Lambda} \lambda(E) \\
= & \sup _{i \in \Lambda}\left\{\sum_{i=0}^{n-2} \lambda\left(E \cap\left(S_{x_{i}^{\prime}}-S_{x_{i}}\right)\right)+\sum_{i=0}^{n-2} \lambda\left(E \cap\left(S_{x_{i+1}}-S_{x_{i}^{\prime}}\right)\right)\right. \\
& \left.\quad+\lambda\left(E \cap\left(\bar{S}_{r x_{0}}-S_{x_{n-1}}\right)\right)\right\} \\
\leqq & \sum_{i=0}^{n-2} \lambda^{*}\left(E \cap\left(S_{x_{i}^{\prime}}-S_{x_{i}}\right)\right)+\sum_{i=0}^{n-2} \lambda^{*}\left(E \cap\left(S_{x_{i+1}}-S_{x_{i}^{\prime}}\right)\right) \\
& +\lambda^{*}\left(E \cap\left(S_{r x_{0}}-S_{x_{n-1}}\right)\right) .
\end{aligned}
$$

(3.10) and (3.11) yield

$$
\lambda^{*}(E)=\lim _{m \rightarrow \infty} \lambda_{1 / m}(E) \quad\left(E \in \mathscr{M}_{0}\right) .
$$

Now, since $\lambda_{1 / m} \in \Lambda$,

$$
\lambda_{1 / m}(E)=\int_{E} f\left(x, \lambda_{1 / m}\left(\bar{S}_{x}\right)\right) d \mu, \quad E \subset S_{r x_{0}}-S_{x_{0}} \quad\left(E \in \mathscr{M}_{0}\right) .
$$

Taking limits as $m \rightarrow \infty$, it follows from (3.12) and conditions (ii) and (iii), using Lebesgue's dominated convergence theorem, that

$$
\lambda^{*}(E)=\int_{E} f\left(x, \lambda^{*}\left(\bar{S}_{x}\right)\right) d \mu \quad \text { for } E \subset S_{r x_{0}}-S_{x_{0}} \quad\left(E \in \mathscr{M}_{0}\right) .
$$

$\lambda^{*}$ is thus a maximum solution of $(*)$.

Similarly, if we define $\lambda_{*}(E)=\inf _{\lambda \in \Lambda}\{\lambda(E)\}\left(E \in \mathscr{M}_{0}\right)$, it can be proved that $\lambda_{*}$ is a minimum solution of $(*)$.

This completes the proof.

\section{REFERENCES}

1. E. A. Coddington and N. Levinson, Theory of ordinary differential equations, McGraw-Hill, New York, 1955. MR 16, 1022.

2. N. Dunford and J. T. Schwartz, Linear operators. I: General theory', Pure and Appl. Math., vol. 7, Interscience, New York, 1958. MR 22 \#8302.

3. R. R. Sharma, An abstract measure differential equation, Proc. Amer. Math. Soc. 32 (1972), 503-510.

Department of Mathematics, Regional Institute of Technology, Jamshedpur, INDIA 